(2) Open Access Full Text Article

\title{
Intensity-modulated radiotherapy for gliomas: dosimetric effects of changes in gross tumor volume on organs at risk and healthy brain tissue
}

This article was published in the following Dove Press journal:

OncoTargets and Therapy

15 June 2016

Number of times this article has been viewed

Zhen Yang'
Zijian Zhang'
Xia Wang'
Yongmei Hu'
Zhiping Lyu'
Lei Huo'
Rui Wei'
Jun Fu'
Jidong Hong'
'Department of Oncology,
'Department of Neurosurgery,
The Institute of Skull Base Surgery
and Neurooncology at Hunan,
Central South University, Xiangya
Hospital, Changsha, Hunan, People's
Republic of China

Republic of China
Correspondence: Jidong Hong Department of Radiation Oncology,

Central South University, Xiangya

Hospital, No 87, Xiangya Road,

Changsha, Hunan 410008 ,

People's Republic of China

Tel +8673189753733

Fax +86 73। 89753336

Email hongjidong1966@|26.com
Aim: The aim of this study was to explore the effects of changes in the gross tumor volume (GTV) on dose distribution in organs at risk (OARs) and healthy brain tissue in patients with gliomas.

Methods: Eleven patients suffering from gliomas with intensity-modulated radiotherapy (IMRT) plans treated with a simultaneous integrated boost technique planned before therapy (initial plans) were prospectively enrolled. At the end of radiotherapy, patients underwent repeat computed tomography and magnetic resonance imaging, and IMRT was replanned. The GTV and dosimetric parameters between the initial and replanned IMRT were compared using the Wilcoxon two-related-sample test, and correlations between the initial GTV and the replanned target volumes were assessed using the bivariate correlation test.

Results: The volume of the residual tumor did not change significantly $(P>0.05)$, the volume of the surgical cavity decreased significantly $(P<0.05)$, and the GTV and target volumes decreased significantly at the end of IMRT (all $P<0.05$ ). The near-maximum dose to OARs and volumes of healthy brain tissue receiving total doses of 10-50 Gy were lower in the replanned IMRT than in the initial IMRT (all $P<0.05$ ). The GTV in the initial plan was significantly positively correlated with the changes in the GTV and planning target volume 1 that occurred during IMRT (all $P<0.05$ ).

Conclusion: The reduction in the GTV in patients with gliomas resulted from shrinkage of the surgical cavity during IMRT, leading to decreased doses to the OARs and healthy brain tissue. Such changes appeared to be most meaningful in patients with large initial GTV values.

Keywords: intensity-modulated radiotherapy, dose distribution, glioma, gross tumor volume, tumor bed

\section{Introduction}

The broad category glioma represents $\sim 28 \%$ of all tumors and $80 \%$ of malignant tumors. Glioblastoma accounts for the majority of gliomas, while astrocytoma and glioblastoma combined account for about three-fourths of all gliomas as defined by CBTRUS. ${ }^{1}$ Radiotherapy (RT) is one of the adjuvant modalities involved in the multimodal treatment of gliomas. ${ }^{2}$ Five previous clinical trials have demonstrated a statistically significant survival benefit from postoperative RT as compared with the supportive care or the administration of different chemotherapy schedules without RT. ${ }^{3}$ Postoperative RT and concurrent and adjuvant chemotherapy with temozolomide remain the standard treatment for patients with glioblastoma. ${ }^{4}$ In the treatment of high-grade gliomas, intensity-modulated RT (IMRT) improves the radiation dose coverage and allows for dose escalation to target volumes while sparing normal structures. ${ }^{5,6}$ Previous research has indicated that the gross 
tumor volume (GTV) after surgery in gliomas will change as time elapses. Champ et $\mathrm{al}^{7}$ have reported that the magnitude of the target volume changed over the period from immediate postoperative magnetic resonance imaging (MRI) to repeat MRI during the day of RT simulation; the study involved 24 patients with high-grade gliomas, where the target volumes were contoured according to the Radiation Therapy Oncology Group guidelines. ${ }^{8}$ GTV1 (fluid-attenuated inversion recovery abnormality and tumor bed) had decreased significantly in the delayed MRI as compared with the immediate postoperative MRI, while GTV2 (contrast-enhanced T1 abnormality and tumor bed) exhibited a nonsignificant increase. Some studies have found changes in tumor volume in high-grade gliomas during conformal RT and IMRT. Tsien et $\mathrm{al}^{9}$ reported that the GTV changed in 19 patients of high-grade glioma based on MRI data obtained at week 3 during RT: two patients had an objective decrease in GTV, 12 patients revealed a slight decrease in rim enhancement or changes in the cystic appearance of the GTV, two patients showed no changes in GTV, and three patients demonstrated an increase in GTV. Shukla et al ${ }^{10}$ analyzed 15 patients with high-grade gliomas treated with conformal RT; tumor volume assessment was based on MRI data at the end of week 5 of treatment. Twelve patients showed a reduction in tumor volume (median, $54.85 \mathrm{~cm}^{3}$ ), of which four had an objective reduction in GTV ( $\geq 50 \%$ ); the other three patients demonstrated an increase in tumor volume (median, $14 \mathrm{~cm}^{3}$ ).

We discovered a trend of decreasing tumor bed size or surgical cavity volume during IMRT in our treatment of patients with glioma, which resulted in a decrease in GTV. If this volume actually decreased, it would affect the radiation dose delivered to organs at risk (OARs) and healthy brain tissue during IMRT. One aim of this study was to evaluate the changes in the volume of the residual tumor and surgical resection cavity with time after IMRT in patients with gliomas and also to evaluate whether there were changes in the GTV. Additionally, changes in the GTV were analyzed to determine how the dose distributions to the OARs and healthy brain tissue may change during IMRT in response to changes in GTV; furthermore, the magnitude of the dosimetric changes to OARs and healthy brain tissue was also determined. We also analyzed the correlations between the GTV and changes in GTV, planning target volume 1 (PTV1), PTV2, and PTV3 during IMRT.

\section{Materials and methods}

\section{Patients' selection}

A total of eleven patients with gliomas were enrolled in this prospective study at a single institution. The study protocol was approved by Medical Ethics Committee of Xiangya Hospital of Centre South University and all participants gave written informed consent to be included in the study. All patients underwent surgery and at least partial tumor resection, and all the lesions were pathologically confirmed as being gliomas according to the World Health Organization 2007 classification of tumors of the central nervous system. ${ }^{11}$ Four patients were diagnosed as grade 2 gliomas, five patients were proved to be grade 3 gliomas, and two patients were confirmed to be grade 4 gliomas. The extent of surgical resection was evaluated by pre- and postoperative MRI. Gross tumor resection (GTR) consisted of complete removal of visible tumor, subtotal resection (STR) consisted of removal of $50 \%-99 \%$ of the tumor volume, and a biopsy indicated resection of $<50 \%$ of the tumor in contrast-enhanced T1-weighted images. All patients received IMRT. Four patients underwent GTR, and seven patients experienced STR. Patients with a grade 3 or 4 glioma received concomitant chemotherapy consisting of daily temozolomide $\left(75 \mathrm{mg} / \mathrm{m}^{2} / \mathrm{d}\right)$ with IMRT and adjuvant chemotherapy consisting of up to six cycles of maintenance temozolomide (150-200 $\mathrm{mg} / \mathrm{m}^{2} / \mathrm{d}$ on days $1-5$ repeated every 28 days). Corticosteroids were not used regularly during the RT of gliomas in our study. Usually, $250 \mathrm{~mL}$ of $20 \%$ mannitol injection was given once a day within the first 3 days after RT, and when headache and vomiting arose in patients, 5-10 mg of dexamethasone was administrated through vein once a day along with mannitol for 3 days.

\section{IMRT plans}

For treatment planning, each patient was immobilized using an individualized thermoplastic cast. For planning, contrast-enhanced computed tomography (CT) with $0.3 \mathrm{~cm}$ slices, gadolinium-enhanced T1-weighted MRI images, and T2-weighted MRI images were obtained; these CT and MRI images were fused to create the initial image in accordance with the International Commission on Radiation Units and Measurements report number $62 .{ }^{12}$ For grades 3 and 4 gliomas, referring to the definition of the target volume given by Stupp et al, ${ }^{13}$ the GTV represented the residual tumor and surgical cavity in contrast-enhanced T1-weighted images in MRI simulation, meantime residual tumor was defined referring to MRI after surgery within 72 hours. The GTV with a $0.5 \mathrm{~cm}$ margin was defined as the PTV1, and the GTV with a $1.5 \mathrm{~cm}$ margin was defined as the clinical tumor volume 2 (CTV2). The CTV2 with a $0.5 \mathrm{~cm}$ margin was defined as the PTV2. The GTV with a $2.5 \mathrm{~cm}$ margin was defined as the CTV3, and the CTV3 with a $0.5 \mathrm{~cm}$ margin was defined as the PTV3. For grade 2 gliomas, according to the European Organization for Research and Treatment 
of Cancer protocol, ${ }^{14}$ the GTV included the residual tumor and surgical cavity based on the region of high intensity in T2-weighted MRI images; the GTV with a $0.5 \mathrm{~cm}$ margin was generated as the PTV1. The GTV with a $1.5 \mathrm{~cm}$ margin was defined as the CTV2, and the CTV2 with a $0.5 \mathrm{~cm}$ margin was defined as the PTV2. Simultaneous integrated boost-IMRT was performed using Varian's Trilogy treatment system (Varian Oncology, Palo Alto, CA, USA) at 2.4-6.86 weeks after surgery. The mean interval time after surgery was 4.43 weeks, and IMRT was usually performed within 5 days after MRI simulation, giving a different subvolume radiation dose; fractions were delivered five times a week to a total of 26-30 fractions. The PTV1 received a dose to target $\left(\mathrm{D}_{\mathrm{T}}\right)$ of 52.08-64.2 Gy delivered in 2.10-2.14 Gy fractions. The $\mathrm{D}_{\mathrm{T}}$ for PTV2 was 48.6-60.2 Gy delivered in 1.8-2.0 Gy fractions, and the $\mathrm{D}_{\mathrm{T}}$ for PTV3 was 48.6-54 Gy delivered in $1.8 \mathrm{~Gy}$ fractions. OARs included the brainstem, the right and left lens, the right and left optic nerves, the optic chiasm, the pituitary gland, the left and right hippocampi, and the left and right cochleas. Dose limitation to OARs was undertaken with reference to the Radiation Therapy Oncology Group 0825 protocol. ${ }^{15}$ Dmaxs delivered to key OARs were as follows: the lens, $7 \mathrm{~Gy}$; the optic nerves, $55 \mathrm{~Gy}$; the optic chiasm, $56 \mathrm{~Gy}$; the pituitary gland, $50 \mathrm{~Gy}$; the brainstem, $60 \mathrm{~Gy}$; and the cochlea, $35 \mathrm{~Gy}$. Healthy brain tissue was defined as the volume of the whole brain minus the PTV2 for grade 2 gliomas or the PTV3 for grades 3 and 4 gliomas. IMRT plans that were designed using the initial images were referred to as the initial IMRT plans.

On the same day at the end of RT, the second planning contrast-enhanced CT scans, the second planning gadolinium-enhanced T1-weighted MRI scans, and the T2-weighted MRI scans were performed with the patient in the same treatment position and secured using the same thermoplastic cast; the resulting images were coregistered to create the replanned image. The residual tumor, the surgical cavity, and the target volume, which were defined in the same manner as in the initial IMRT plan, were contoured by the same radiation oncologist and neuroradiologist who defined them in the initial IMRT images. The IMRT plans were then designed using the replanned images (IMRT replan) by the same radiation physicist who designed the initial IMRT plans. Both the initial IMRT plans and the IMRT replans were designed using the Eclipse Treatment Planning System (Varian Medical Systems Inc., Palo Alto, CA, USA); the prescribed dose, the plan objective, and the OAR constraints were the same in the IMRT replan as in the initial IMRT plan.

\section{Target volume measurements and dose calculations in OARs and healthy brain tissue}

The volumes of the residual tumor and the surgical cavity, the GTV, and the target volumes, including the PTV1, PTV2, and PTV3, were measured and compared between the initial and replanned images. Dose-volume histograms (DVHs) were generated to determine the near-maximum dose (D2\%) and the mean absorbed dose (D50\%) to the OARs in the initial IMRT plan and in the IMRT replan. For each OAR, a mean DVH used for comparison between the initial and replanned IMRT was obtained by summing its DVH in each plan and averaged by the number of selected patients. Values of the relative volume of healthy brain tissue receiving at least nGy (Vn [\%]) in the initial IMRT plan were compared to those in the IMRT replan using the DVHs for healthy brain tissue. The correlations between the GTV in the initial IMRT plan and the changes in the GTV, PTV1, PTV2, and PTV3 at the end of IMRT were investigated.

\section{Statistical analysis}

Statistical Package for the Social Sciences 13.0 was used to carry out the statistical analysis. Differences in the volumes of the residual tumor and the surgical cavity, the GTV, target volumes, and dosimetric parameters in the OARs and healthy brain tissue between the initial IMRT plan and the IMRT replan were performed using the Wilcoxon tworelated-sample test. The independence of correlated data was the GTV in the initial IMRT, and the dependence of correlated data was changes in the GTV, PTV1, PTV2, and PTV3 at the end of IMRT. Correlations between the GTV in the initial IMRT plan with changes in the GTV, PTV1, PTV2, and PTV3 at the end of IMRT were analyzed using the bivariate correlation tests with Pearson's correlation coefficients. $P$-value of $<0.05$ was considered as statistically significant.

\section{Results}

\section{Comparisons between tumor volumes and target volumes in the initial and replanned IMRT}

There was no significant difference in changes in the volume of the residual tumor between the initial IMRT plan and the IMRT replan (mean reduction, $3.2 \pm 8.2 \mathrm{~cm}^{3} ; P=0.093$ ). In one patient, the volume of the residual tumor after replanning increased as compared to that in the initial plan; in six patients, the volume of the residual tumor decreased 


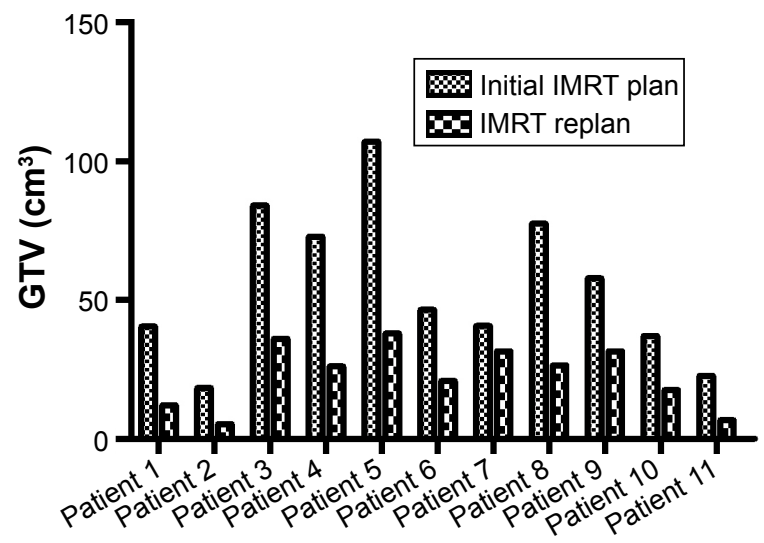

Figure I The changes in the GTV in the initial and replanned IMRT. Abbreviations: GTV, gross tumor volume; IMRT, intensity-modulated radiotherapy.

during IMRT; and in four patients with GTR, there was no new lesion around the resection cavity at the end of IMRT. The surgical cavity, GTV, PTV1, PTV2, and PTV3 values were significantly lower after IMRT replanning than in the initial IMRT plans (mean reductions, $28.91 \pm 14.97 \mathrm{~cm}^{3}$, $31.29 \pm 18.93 \mathrm{~cm}^{3}, 45.2 \pm 22.5 \mathrm{~cm}^{3}, 87.1 \pm 32.3 \mathrm{~cm}^{3}$, and $103.8 \pm 40.3 \mathrm{~cm}^{3}$, respectively; all $P<0.05$ ). All patients showed an objective decrease in the volume of the surgical cavity: nine patients exhibited a reduction in the volume of the surgical cavity of $\geq 50 \%$ and two patients demonstrated a reduction in the volume of the surgical cavity of $<50 \%$. The GTV, PTV1, PTV2, and PTV3 of the initial contrastenhanced T1-weighted MRI images in patients with grades 3 and 4 gliomas and the T2-weighted MRI images in patients with grade 2 gliomas objectively decreased relative to those in the replanned contrast-enhanced T1- and T2-weighted MRI images. Figure 1 shows the changes in GTV in the initial and replanned IMRT. The changes in GTV between the initial and replanned MRI images for patient 4 are shown in Figure 2. The clinical data of patients are shown in Table 1. The values of tumor volume in the initial and replanned IMRT in each patient are shown in Table 2. Changes in the residual tumor volumes and surgical cavity, GTV, and target volumes between the initial and replanned IMRT are shown in Table 3.

\section{Comparison between dosimetric parameters of OARs and healthy brain tissue in the initial IMRT plan and the IMRT replan}

Values for dosimetric parameters regarding the OARs and healthy brain tissue were acquired from the DVHs for the
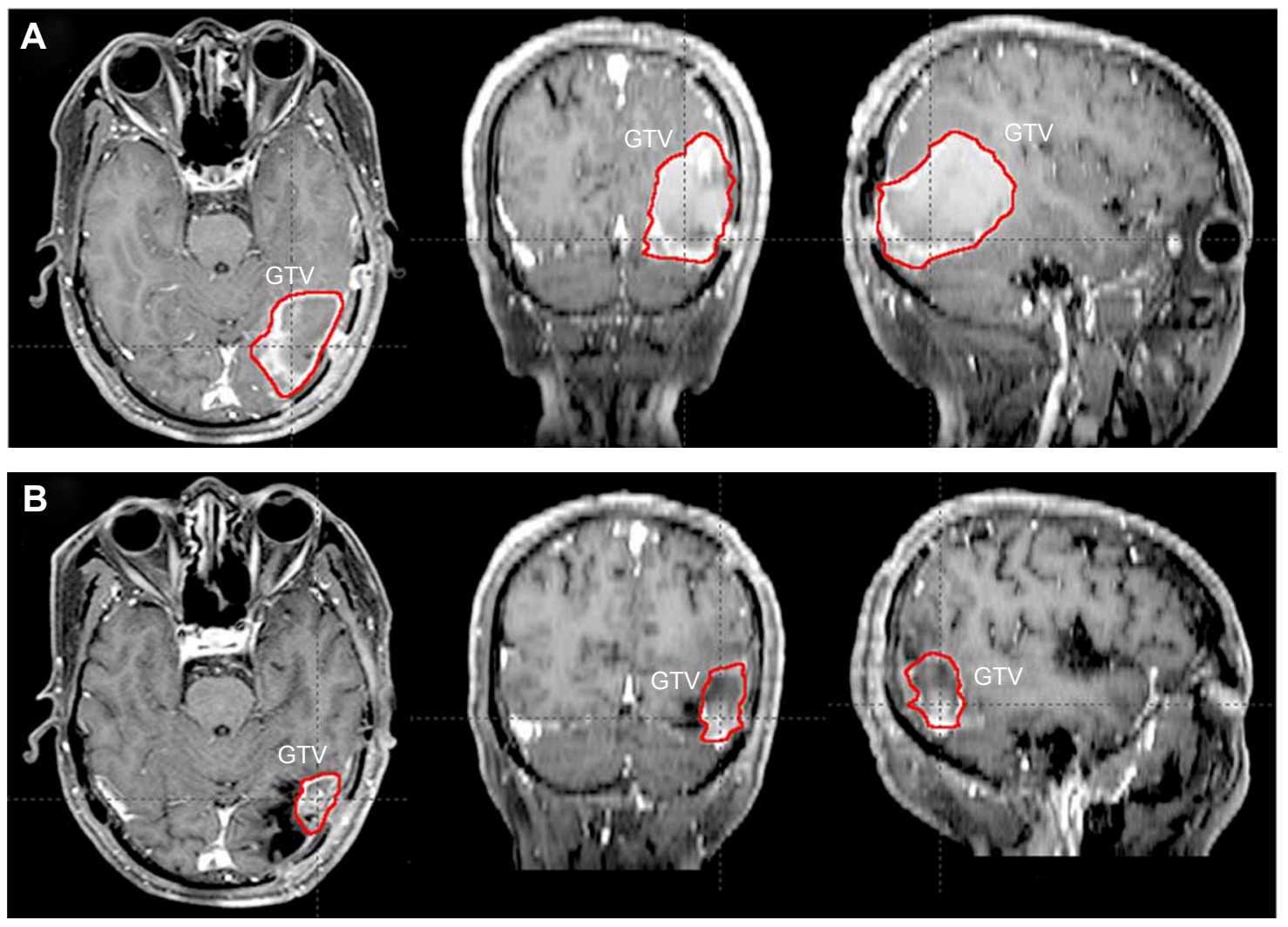

Figure 2 GTV contours for patient 4 in the (A) initial IMRT plan and (B) replanned IMRT. Abbreviations: GTV, gross tumor volume; IMRT, intensity-modulated radiotherapy. 
Table I Clinical characteristics of eleven patients with gliomas

\begin{tabular}{|c|c|c|c|c|c|c|c|}
\hline \multirow{2}{*}{$\begin{array}{l}\text { Patient } \\
\text { number }\end{array}$} & \multirow[t]{2}{*}{ Side } & \multirow[t]{2}{*}{ Location of tumor } & \multirow{2}{*}{$\begin{array}{l}\text { Extent of surgical } \\
\text { resection }\end{array}$} & \multirow[t]{2}{*}{ Pathological grade } & \multicolumn{3}{|c|}{ Radiation dose (Gy) } \\
\hline & & & & & PTVI & PTV2 & PTV3 \\
\hline I & Left & Frontal lobe & STR & 3 & 64.2 & 60 & 54 \\
\hline 2 & Left & Frontal lobe & GTR & 3 & 64.2 & 60 & 54 \\
\hline 3 & Left & Frontal lobe & STR & 4 & 64.2 & 60 & 54 \\
\hline 4 & Left & $\begin{array}{l}\text { Temporal and } \\
\text { occipital lobes }\end{array}$ & STR & 4 & 64.2 & 60 & 54 \\
\hline 5 & Right & $\begin{array}{l}\text { Frontal and parietal } \\
\text { lobes }\end{array}$ & STR & 2 & 57.78 & 48.6 & \\
\hline 6 & Left & Frontal lobe & GTR & 2 & 54 & 48.6 & \\
\hline 7 & Right & Occipital lobe & STR & 2 & 52.08 & 50.4 & \\
\hline 8 & Right & $\begin{array}{l}\text { Temporal and } \\
\text { parietal lobes }\end{array}$ & GTR & 3 & 60.2 & 60.2 & 54 \\
\hline 9 & Right & Frontal lobe & GTR & 3 & 60 & 54 & 46.8 \\
\hline 10 & Right & Frontal lobe & STR & 2 & 58.8 & 50.96 & \\
\hline II & Right & Parietal lobe & STR & 3 & 60.2 & 60.2 & 54 \\
\hline
\end{tabular}

Abbreviations: GTR, gross tumor resection; PTVI, planning target volume I; PTV2, planning target volume 2; PTV3, planning target volume 3; STR, subtotal resection.

initial IMRT plan and the IMRT replan. The mean DVHs for OARs in the two sets of plans are displayed in Figure 3. $\mathrm{D} 2 \%$ to the brainstem, optic chiasma, and pituitary gland in the IMRT replan was lower than that in the initial IMRT plan (mean reductions, $8.0 \pm 2.8 \mathrm{~Gy}, 7.9 \pm 3.4 \mathrm{~Gy}$, and $5.7 \pm 2.6 \mathrm{~Gy}$, respectively; all $P<0.05$ ). D2\% to the ipsilateral lens, contralateral lens, ipsilateral optic nerve, and contralateral optic nerve in the IMRT replans were significantly lower than those in the initial IMRT plans (mean reductions, $1.0 \pm 0.2 \mathrm{~Gy}$, $0.7 \pm 0.3 \mathrm{~Gy}, 3.8 \pm 1.4 \mathrm{~Gy}$, and 5.0 $\pm 1.9 \mathrm{~Gy}$, respectively; all $P<0.05)$. Similarly, D2\% to the ipsilateral hippocampus, contralateral hippocampus, ipsilateral cochlea, and contralateral cochlea in the IMRT replan was significantly lower than that in the initial IMRT plan (mean reductions, $6.8 \pm 2.2 \mathrm{~Gy}$, 13.8 $\pm 6.4 \mathrm{~Gy}, 9.5 \pm 3.8 \mathrm{~Gy}$, and 5.2 $\pm 2.2 \mathrm{~Gy}$, respectively; all $P<0.05)$. D50\% to the ipsilateral hippocampus, contralateral hippocampus, ipsilateral cochlea, and contralateral cochlea in the IMRT replan was significantly lower than that in the initial IMRT plan (mean reductions, 5.8 $\pm 3.1 \mathrm{~Gy}, 8.1 \pm 5.6 \mathrm{~Gy}$, $6.6 \pm 4.9 \mathrm{~Gy}$, and $4.5 \pm 3.7 \mathrm{~Gy}$, respectively; all $P<0.05$ ). V10, $\mathrm{V} 20, \mathrm{~V} 30, \mathrm{~V} 40$, and V50 values in the IMRT replan were also lower than those in the initial IMRT plan (mean reductions, $11.3 \% \pm 8.1 \%, 10.9 \% \pm 8.8 \%, 8.9 \% \pm 8.4 \%, 6.5 \% \pm 3.8 \%$, and $3.1 \% \pm 2.5 \%$, respectively; all $P<0.05$ ). Dose distributions for the OARs and the healthy brain tissue in the initial and replanned IMRT of patient 4 are shown in Figure 4. The values of dosimetric parameters for the OARs and the healthy brain tissue are shown in Table 3.

\section{Correlation between the GTV in the initial IMRT plan and changes in the target volumes at the end of IMRT}

The GTV in the initial IMRT plan was positively correlated with changes in the GTV and the PTV1 at the end of IMRT ( $r=0.930$ with $P=0.000$ and $r=0.850$ with $P=0.001$, respectively). There were no correlations between the GTV in

Table 2 The values of tumor volume in the initial and replanned IMRT in each patient

\begin{tabular}{|c|c|c|c|c|c|c|}
\hline \multirow{2}{*}{$\begin{array}{l}\text { Patient } \\
\text { number }\end{array}$} & \multicolumn{3}{|c|}{ Initial IMRT target volume $\left(\mathrm{cm}^{3}\right)$} & \multicolumn{3}{|c|}{ Replanned IMRT target volume $\left(\mathrm{cm}^{3}\right)$} \\
\hline & Residual tumor & Surgical cavity & GTV & Residual tumor & Surgical cavity & GTV \\
\hline 1 & 5.2 & 35.2 & 40.4 & 4.5 & 7.3 & 12.0 \\
\hline 2 & 0 & 18.3 & 18.3 & 0 & 5.3 & 5.3 \\
\hline 3 & 17.9 & 66.2 & 84.1 & 15.9 & 20.4 & 36.2 \\
\hline 4 & 1.1 & 71.7 & 72.8 & 0.0 & 26.1 & 26.1 \\
\hline 5 & 49.4 & 57.6 & 107.0 & 21.9 & 16.0 & 37.9 \\
\hline 6 & 0 & 46.4 & 46.4 & 0 & 20.8 & 20.8 \\
\hline 7 & 0 & 40.7 & 40.7 & 0 & 31.6 & 31.6 \\
\hline 8 & 0 & 77.6 & 77.6 & 0 & 26.4 & 26.4 \\
\hline 9 & 0 & 57.8 & 57.8 & 0 & 36.1 & 36.1 \\
\hline 10 & 0.9 & 36.0 & 37.0 & 2.1 & 15.5 & 17.5 \\
\hline 11 & 6.9 & 15.8 & 22.7 & 1.3 & 5.4 & 6.7 \\
\hline
\end{tabular}

Abbreviations: GTV, gross tumor volume; IMRT, intensity-modulated radiotherapy. 
Table 3 Comparison target volume and dose parameter of OARs in the initial and replanned IMRT

\begin{tabular}{|c|c|c|c|c|c|}
\hline & $\begin{array}{l}\text { Initial IMRT } \\
(\text { mean } \pm \text { SD) }\end{array}$ & $\begin{array}{l}\text { Replanned IMRT } \\
(\text { mean } \pm \text { SD) }\end{array}$ & $\begin{array}{l}\text { Decreased percent during } \\
\text { IMRT (mean } \pm \text { SD) }\end{array}$ & $\mathbf{Z}$ & $P$-value \\
\hline \multicolumn{6}{|l|}{ Target volume $\left(\mathrm{cm}^{3}\right)$} \\
\hline Residual tumor & $7.4 \pm 14.9$ & $4.2 \pm 7.5$ & $14.3 \pm 54.5$ & -1.682 & 0.093 \\
\hline Surgical cavity & $47.6 \pm 20.6$ & $19.2 \pm 10.4$ & $61.1 \pm 14.8$ & -2.934 & 0.003 \\
\hline GTV & $55.0 \pm 27.5$ & $23.5 \pm 11.8$ & $57.1 \pm 15.3$ & -2.934 & 0.003 \\
\hline PTVI & $|04.4 \pm 4| .4$ & $59.3 \pm 24.6$ & $43.0 \pm 12.8$ & -2.943 & 0.003 \\
\hline PTV2 & $301.8 \pm 75.6$ & $2 \mid 4.7 \pm 51.2$ & $28.3 \pm 7.2$ & -2.943 & 0.003 \\
\hline PTV3 & $439.0 \pm 76.7$ & $335.2 \pm 5 I .1$ & $23.2 \pm 7.3$ & -2.201 & 0.028 \\
\hline \multicolumn{6}{|l|}{ D2\% to OARs (Gy) } \\
\hline Brainstem & $46.6 \pm 11.1$ & $38.6 \pm 13.8$ & $20.6 \pm 13.1$ & -2.845 & 0.004 \\
\hline Optic chiasma & $35.3 \pm 12.5$ & $27.4 \pm 9.0$ & $23.8 \pm 18.4$ & -2.667 & 0.008 \\
\hline Pituitary gland & $27.8 \pm 7.2$ & $22.1 \pm 8.6$ & $30.4 \pm 23.1$ & -2.756 & 0.006 \\
\hline Ipsilateral lens & $5.1 \pm 2.3$ & $4.3 \pm 1.1$ & $20.4 \pm 15.3$ & -2.580 & 0.010 \\
\hline Contralateral lens & $4.4 \pm I .5$ & $3.6 \pm 1.3$ & $18.2 \pm 15.5$ & -2.402 & 0.016 \\
\hline Ipsilateral optic nerve & $22.3 \pm 8.3$ & $18.4 \pm 7.2$ & $24.7 \pm 18.6$ & -2.934 & 0.003 \\
\hline Contralateral optic nerve & $21.8 \pm 7.5$ & $16.8 \pm 6.3$ & $33.8 \pm 22.7$ & -2.934 & 0.003 \\
\hline Ipsilateral hippocampus & $51.5 \pm 14.1$ & $44.6 \pm 17.4$ & $15.7 \pm 12.8$ & -2.667 & 0.008 \\
\hline Contralateral hippocampus & $42.6 \pm 15.2$ & $28.8 \pm 14.8$ & $33.3 \pm 19.4$ & -2.845 & 0.004 \\
\hline Ipsilateral cochlea & $24.6 \pm 9.3$ & $|5| \pm 5.8$. & $37.7 \pm 32.0$ & -2.758 & 0.010 \\
\hline Contralateral cochlea & $13.4 \pm 4.2$ & $8.2 \pm 4.0$ & $30.2 \pm 24.8$ & -2.490 & 0.013 \\
\hline \multicolumn{6}{|l|}{ D50\% to OARs (Gy) } \\
\hline Ipsilateral hippocampus & $44.1 \pm 18.1$ & $38.3 \pm 18.6$ & $17.8 \pm 15.7$ & -2.802 & 0.005 \\
\hline Contralateral hippocampus & $28.2 \pm 15.6$ & $20.11 \pm 13.7$ & $28.9 \pm 15.5$ & -2.845 & 0.004 \\
\hline Ipsilateral cochlea & $16.8 \pm 8.2$ & $10.2 \pm 4.9$ & $39.7 \pm 25.9$ & -2.845 & 0.004 \\
\hline Contralateral cochlea & $10.7 \pm 3.5$ & $6.2 \pm 3.7$ & $35.2 \pm 23.4$ & -2.934 & 0.003 \\
\hline \multicolumn{6}{|l|}{ Vn (\%) of healthy brain } \\
\hline Vio & $72.6 \pm 11.0$ & $61.2 \pm 12.7$ & $|5.7 \pm| \mid .3$ & -2.576 & 0.006 \\
\hline V20 & $50.8 \pm 10.0$ & $39.9 \pm 10.2$ & $20.8 \pm 17.2$ & -2.576 & 0.006 \\
\hline V30 & $32.1 \pm 9.0$ & $23.3 \pm 5.7$ & $26.0 \pm 14.4$ & -2.845 & 0.004 \\
\hline V40 & $16.8 \pm 6.1$ & $10.3 \pm 3.0$ & $35.2 \pm 15.1$ & -2.845 & 0.004 \\
\hline V50 & $5.2 \pm 3.8$ & $2.1 \pm 1.6$ & $52.5 \pm 24.9$ & -2.803 & 0.005 \\
\hline
\end{tabular}

Notes: D2\%: near-maximum dose; D50\%: median absorbed dose; and $\mathrm{Vn}$ (\%): values of the relative volume of healthy brain tissue receiving at least $\mathrm{nGy}$. $Z$ is the statistic of the Wilcoxon two-related-sample test.

Abbreviations: GTV, gross tumor volume; IMRT, intensity-modulated radiotherapy; OARs, organs at risk; PTVI, planning target volume I; PTV2, planning target volume 2; PTV3, planning target volume 3 .

the initial plan and changes in the PTV2 and PTV3 ( $r=0.501$ with $P=0.117$ and $r=0.668$ with $P=0.147$, respectively). Because of the small sample size, correlation between GTV in the initial IMRT plan and changes in the PTV2 and PTV3 during IMRT was not statistically significant $(P>0.05)$. However, correlation between GTV and changes in the PTV2 and PTV3 was $r>0.5$. If the sample was big enough, these correlations may be statistically significant, even if the $r$ value remains the same level. Figure 5 shows the correlation between the GTV in the initial IMRT plan and the changes in the GTV and PTV1 at the end of IMRT.

\section{Discussion}

The patients enrolled in the study were not consecutive cases. To explore the effects of changes in the GTV of the residual tumor and the surgical cavity during IMRT for gliomas, we selected patients with gliomas who had undergone GTR or STR with an obvious surgical cavity from January 2009 to January 2012. We carried out the IMRT replan, collected the data, and performed the statistical analysis from February 2012 to October 2012. This study confirmed that the GTV values for gliomas declined between the initial IMRT plan and the end of IMRT; there was a reduction in the PTV1, PTV2, and PTV3 at the end of IMRT. These volume changes were associated with decreases in the D2\% and D50\% values for the OARs. We also observed that there were positive correlations between the GTV value in the initial IMRT plan and changes in the GTV and PTV1 values at the end of IMRT. Two studies have evaluated changes in the volume of the GTV during RT in high-grade gliomas. Tsien et $\mathrm{al}^{9}$ reported that the GTV changed in 19 patients with high-grade gliomas based on MRI data obtained early during RT. Most patients exhibited an 

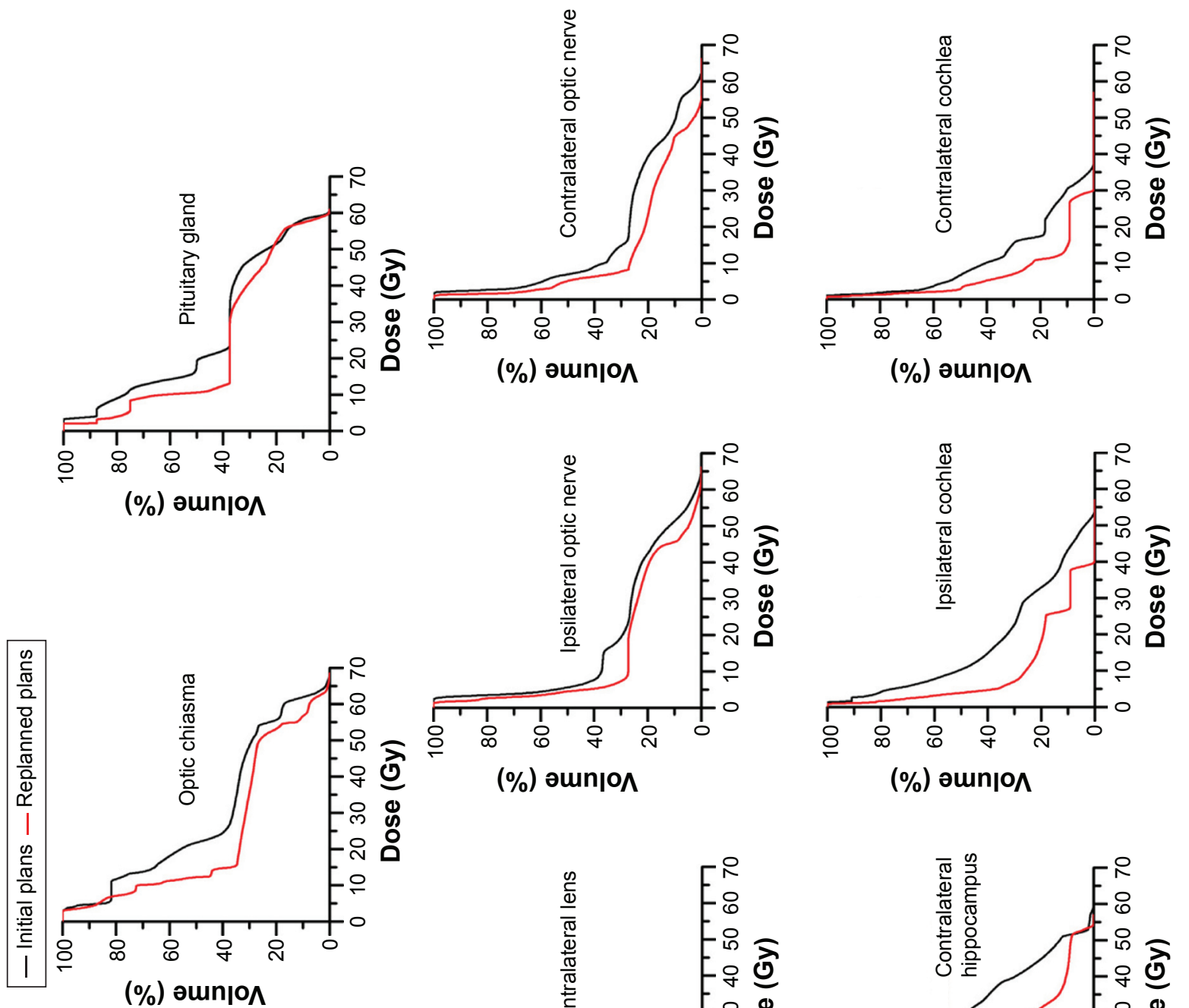

(\%) әшn|о

(\%) әun|o^
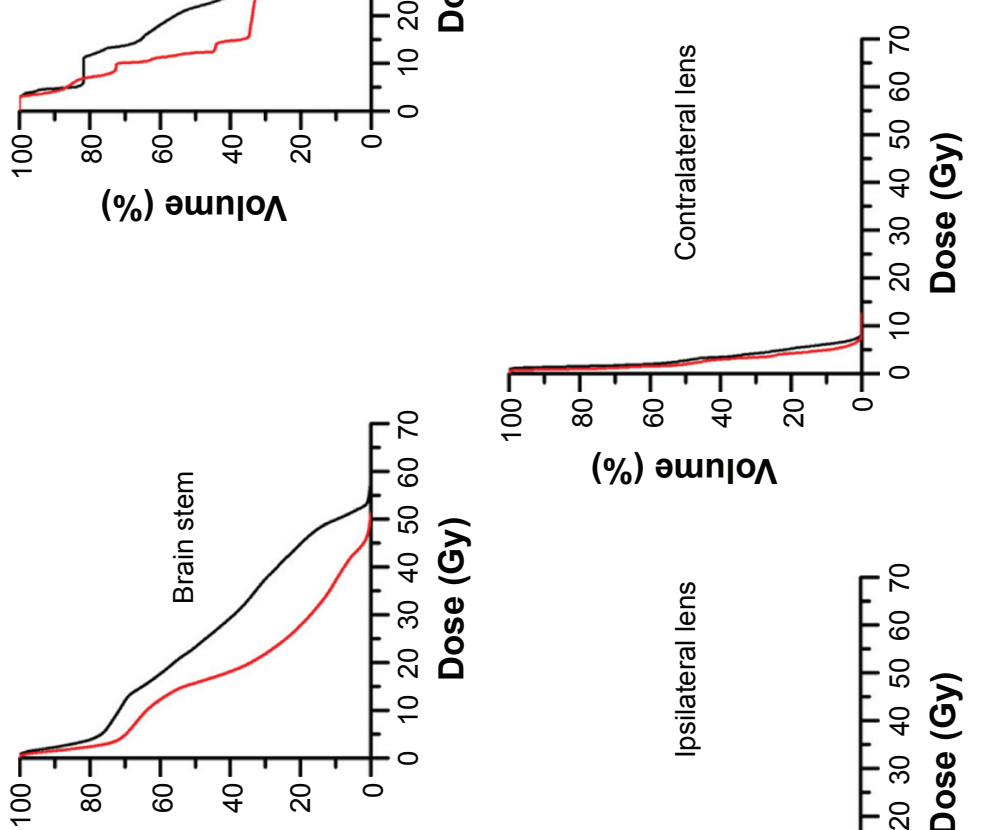

(\%) əunnjo

(\%) əun|o

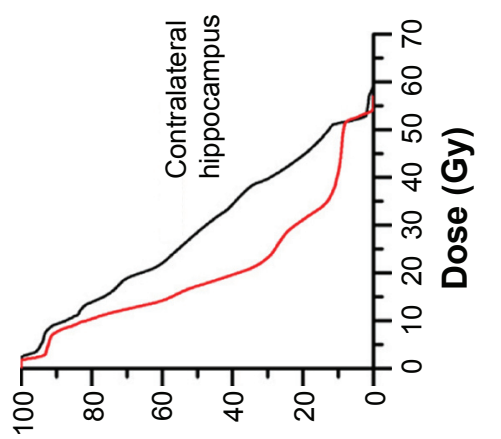

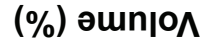

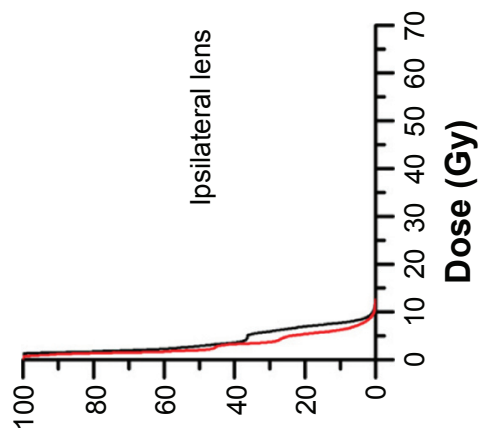

(\%) әயn|о^

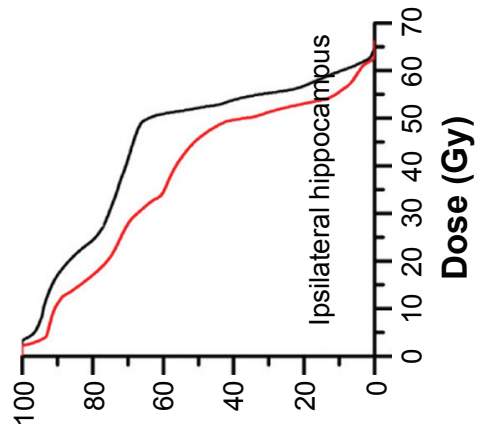

(\%) əunno^ 

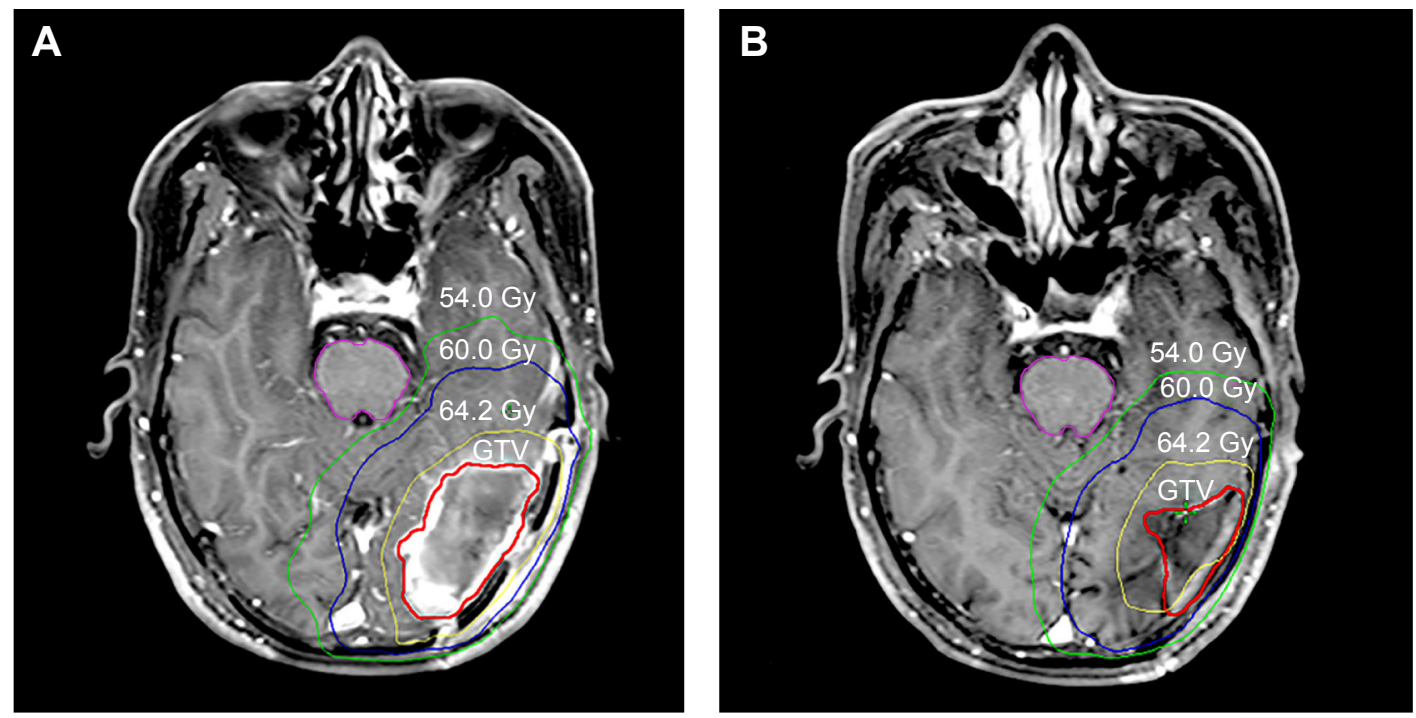

Figure 4 Dose distributions in GTV and OARs in the (A) initial and (B) replanned IMRT plans for patient 4. Abbreviations: GTV, gross tumor volume; IMRT, intensity-modulated radiotherapy; OARs, organs at risk.

objective or slight decrease in the GTV; in two patients, there were no changes in the GTV, and in three patients, there was an increase in the GTV. Both of the patients with an objective decrease in the GTV during treatment involved grade 3 tumors. No cases of tumor progression were noted in patients with grade 3 tumors during treatment. In comparison, three of the 12 patients with grade 4 tumors exhibited tumor progression, based on the MRI scans obtained during week 3 of RT. This study found that the GTV in the replanned MRI image in the total patient population objectively decreased relative to the GTV in the initial MRI image, which differed from the findings of Tsien et al. ${ }^{9}$ This study included four patients with grade 2 gliomas, five patients with grade 3 gliomas, and two patients with grade 4 gliomas. Perhaps the changes in the GTV were relative to the grade of the gliomas. The high-grade gliomas were more aggressive than the low-grade gliomas, and the
A

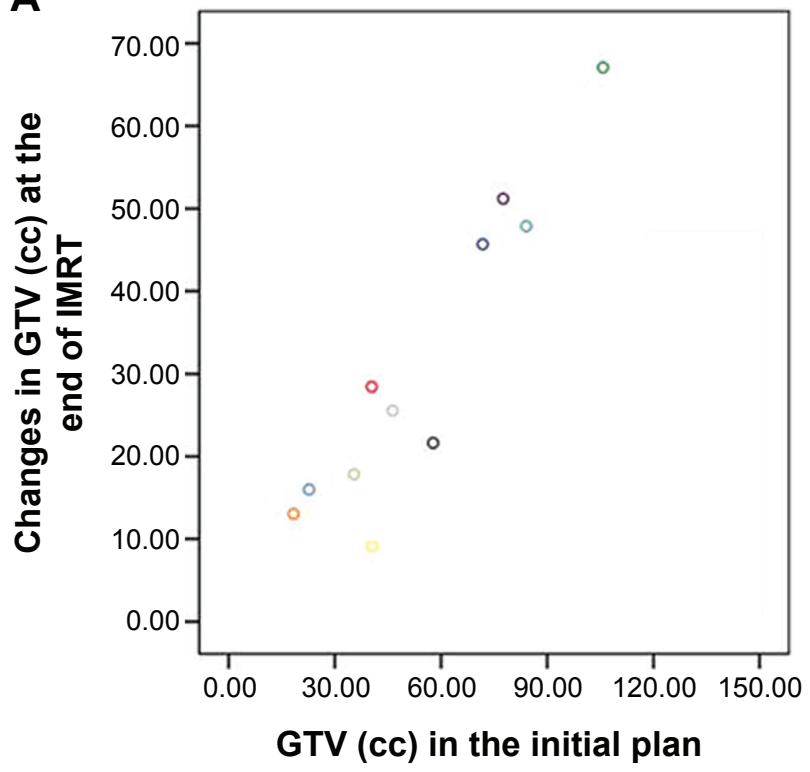

B

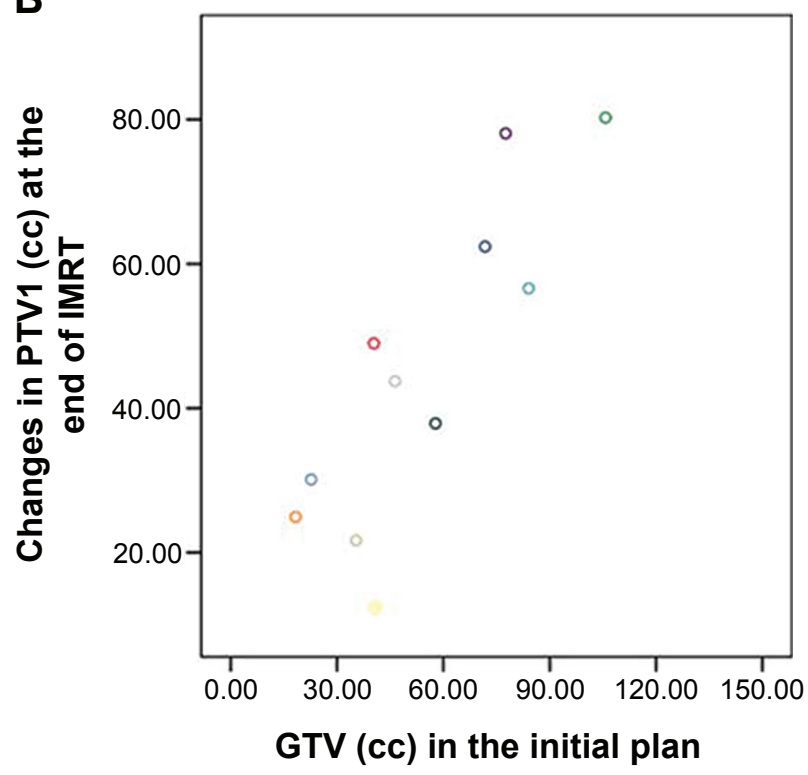

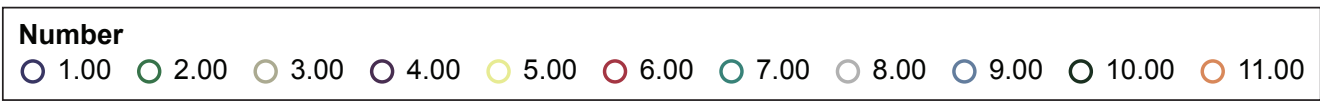

Figure 5 Correlation between the GTV in the initial plan and the changes in the GTV at the end of IMRT (A) and the changes in the PTVI at the end of IMRT (B). Abbreviations: GTV, gross tumor volume; IMRT, intensity-modulated radiotherapy; PTVI, planning target volume I. 
probability of progressive disease in the high-grade gliomas during RT may be higher than that in the low-grade gliomas. The extent of surgery also differed between the two studies. In this study, all patients underwent GTR or STR. However, in the study of Tsien et al, ${ }^{9}$ six patients received STR and seven patients underwent biopsy. Local control was associated with the extent of resection. Patients who had received GTR and STR had bigger surgical cavities than patients who had received STR and biopsy. The residual tumor and surgical cavity constituted the GTV. In this study, there was no significant difference in the changes in the volumes of the residual tumor between the initial IMRT plan and the IMRT replan. The surgical cavity was significantly smaller in the IMRT replan than in the initial IMRT plan. The shrinkage of the surgical cavity resulted in a decrease in the GTV. Shukla et $\mathrm{al}^{10}$ evaluated the changes in the GTV in 17 patients with high-grade gliomas at the end of week 5 of conformal RT. Fifteen patients received STR, two patients experienced biopsy, and two patients with multifocal lesions were excluded from the final analysis. Three patients (two patients with grade 4 and one patient with grade 3 gliomas) exhibited an increase in the GTV, which differed from our findings. We considered that the different extents of resection and the different tumor grades of patients in the two studies lead to differences in the research findings. In our clinical practice, we always found the GTV enlarged at the end of IMRT compared to that at the beginning of IMRT in gliomas; however, in the eleven patients of this study, we did not observe a larger GTV at the end of IMRT than that at the beginning of IMRT. The possible explanation is that the sample size in this study is small.

The change of surgical cavities is related to the time elapsed from surgery to the time MRI was performed. In this study, the interval of the surgery and IMRT was similar to that of the study by Stupp et al, ${ }^{4}$ in which a median of 5 weeks was reported; the mean interval time between surgery and IMRT in our study was 4.43 weeks. In the study of Tsien et al, ${ }^{9}$ simulated T1-enhanced MRI was performed 1-2 weeks before the start of RT, and two additional scans were performed during RT (weeks 1 and 3) and compared the changes in the GTV in week 1 and week 3. The median time interval between surgical resection and pre-RT MRI was 3.1 weeks (range, 2.1-3.8 weeks). Adding the 1-2 weeks interval before RT, the time between the MRI and RT simulations was probably 5 weeks, which was similar to that in this study, so the changes in the GTV were comparable with our result. However, the observed time point was different during RT (week 1 and week 3 ) compared to that in our study (beginning of IMRT and end of IMRT).

In the research by Champ et al, ${ }^{7}$ an average of 17 days elapsed between immediate MRI within 48 hours postoperation and delayed MRI during the day of RT simulation. For treatment planning, often occurring 2-4 weeks after craniotomy, 24 patients were involved, in which 12 patients were given dexamethasone treatment at the time of MRI. Champ et $\mathrm{al}^{7}$ compared the changes in the GTV at 48 hours postoperation compared to that on the day of RT simulation before RT. Our study evaluated the changes in the GTV during RT and prophylactically used mannitol and/or dexamethasone at the beginning of IMRT within the first 3 days. In our research, besides mannitol and/or dexamethasone being an influence on GTV, IMRT and temozolomide had effect on the changes in the GTV.

However, our study had a number of limitations. For instance, the results from our small sample should be validated in larger studies. Additionally, future studies should be designed to accommodate $\mathrm{CT}$ and/or MRI at more time points during therapy and to clarify when changes in the GTV begin to have significant effects on dose distributions in OARs and healthy brain tissue.

\section{Conclusion}

On the basis of our data, we concluded that shrinkage of the surgical cavity or tumor bed caused the reduction in the GTV value during the course of IMRT. Changes in the residual tumor itself may also influence the GTV value in gliomas. In this study, one of the eleven patients exhibited an enlarged residual tumor during IMRT, and in the remaining patients, the residual tumors had decreased in volume at the end of IMRT relative to the volumes before IMRT. Because of the objective shrinkage of the surgical cavity in all patients, the GTV decreased during IMRT. We believed that this decrease in GTV was largely caused by shrinkage of the resection cavity. This study reveals the dosimetric consequences of administering RT exclusively using the initial treatment plan throughout the course of therapy. It resulted in increases in both the D2\% and D50\% delivered to the OARs and the volumes of normal tissue that were irradiated. We observed that the GTV decreased during RT for gliomas; this decrease resulted in decreases in the D2\% and D50\% to the OARs and the $\mathrm{Vn}(\%)$ of healthy brain tissue. Additionally, we found a positive correlation between the initial GTV and shrinkage changes in the GTV and PTV1 at the end of IMRT. The explanation to our study must be very cautious. Gliomas, however, have no distinct border and infiltrate well outside the obvious tumor or surgical cavity, especially in high-grade gliomas, The definition of CTV in gliomas is difficult; $~ 80 \%$ of subclinical lesions exist in the $2 \mathrm{~cm}$ margin around the GTV of malignant gliomas, $90 \%-100 \%$ of tumor relapse occurs within $2 \mathrm{~cm}$ of the enhancing rim when doses in the range of 60-90 Gy are 
given, ${ }^{16-18}$ and the gliomas are resistant to the radiation. If we decreased the CTV of gliomas according to the shrinkage of the surgical cavity in the late stage of IMRT, this most likely will result in a more rapid development of recurrent tumor. For patients with larger GTVs, especially for patients with a bigger surgical cavity, the mid-IMRT CT and MRI simulations are scanned during the later stages of the IMRT course, and the IMRT is replanned to escalate the dose to the GTV while sparing the OARs and healthy brain tissue. It remains uncertain as to whether or not the replanning of IMRT can improve the local control and the long-term survival of patients with gliomas. Studies on adaptive RT for gliomas are needed to improve the local control and the overall survival and to decrease the radiation toxicity for gliomas in the future.

\section{Acknowledgments}

This study was supported by Hunan Province Development and Reform Committee Science Research Fund (2010-1060 and 2014-463), Hunan Province Science and Technology Program (2011SK3223 and 2011SK3220), Hunan Provincial Natural Science Foundation of China (2012JJ5043), and neurooncology research project, Chinese Society of Neurooncology (CNSO-2014-MSD14).

\section{Disclosure}

The authors report no conflicts of interest in this work.

\section{References}

1. Ostrom QT, Gittleman H, Farah P, et al. CBTRUS statistical report: primary brain and central nervous system tumors diagnosed in the United States in 2006-2010. Neuro Oncol. 2013;15(Suppl 2):ii1-ii56.

2. Wind JJ, Young R, Saini A, Sherman JH. The role of adjuvant radiation therapy in the management of high-grade gliomas. Neurosurg Clin N Am. 2012;23(2):247-258.

3. Laperriere N, Zuraw L, Cairncross G. Radiotherapy for newly diagnosed malignant glioma in adults: a systematic review. Radiother Oncol. 2002; 64(3):259-273.

4. Stupp R, Hegi ME, Mason WP, et al; European Organisation for Research and Treatment of Cancer Brain Tumour and Radiation Oncology Groups; National Cancer Institute of Canada Clinical Trials Group. Effects of radiotherapy with concomitant and adjuvant temozolomide versus radiotherapy alone on survival in glioblastoma in a randomised phase III study: 5-year analysis of the EORTC-NCIC trial. Lancet Oncol. 2009;10(5):459-466.

OncoTargets and Therapy

\section{Publish your work in this journal}

OncoTargets and Therapy is an international, peer-reviewed, open access journal focusing on the pathological basis of all cancers, potential targets for therapy and treatment protocols employed to improve the management of cancer patients. The journal also focuses on the impact of management programs and new therapeutic agents and protocols on

Submit your manuscript here: http://www.dovepress.com/oncotargets-and-therapy-journal
5. MacDonald SM, Ahmad S, Kachris S, et al. Intensity modulated radiation therapy versus three-dimensional conformal radiation therapy for the treatment of high grade glioma: a dosimetric comparison. J Appl Clin Med Phys. 2007;8(2):47-60.

6. Tsien CI, Brown D, Normolle D, et al. Concurrent temozolomide and dose-escalated intensity-modulated radiation therapy in newly diagnosed glioblastoma. Clin Cancer Res. 2012;18(1):273-279.

7. Champ CE, Siglin J, Mishra MV, et al. Evaluating changes in radiation treatment volumes from post-operative to same-day planning MRI in High-grade gliomas. Radiat Oncol. 2012;7:220.

8. Chinnaiyan P, Won M, Wen PY, et al. RTOG 0913: a phase 1 study of daily everolimus (RAD001) in combination with radiation therapy and temozolomide in patients with newly diagnosed glioblastoma. Int J Radiat Oncol Biol Phys. 2013;86(5):880-884.

9. Tsien C, Gomez-Hassan D, Ten Haken RK, et al. Evaluating changes in tumor volume using magnetic resonance imaging during the course of radiotherapy treatment of high-grade gliomas: implications for conformal dose-escalation studies. Int J Radiat Oncol Biol Phys. 2005; 62(2):328-332.

10. Shukla D, Huilgol NG, Trivedi N, Mekala C. T2 weighted MRI in assessment of volume changes during radiotherapy of high grade gliomas. J Cancer Res Ther. 2005;1(4):235-238.

11. Louis DN, Ohgaki H, Wiestler OD, et al. The 2007 WHO classification of tumours of the central nervous system. Acta Neuropathol. 2007; 114(2):97-109.

12. Stroom JC, Heijmen BJ. Geometrical uncertainties, radiotherapy planning margins, and the ICRU-62 report. Radiother Oncol. 2002; 64(1):75-83.

13. Stupp R, Mason WP, van den Bent MJ, et al; European Organisation for Research and Treatment of Cancer Brain Tumor and Radiotherapy Groups; National Cancer Institute of Canada Clinical Trials Group. Radiotherapy plus concomitant and adjuvant temozolomide for glioblastoma. N Engl J Med. 2005;352(10):987-996.

14. Musat E, Roelofs E, Bar-Deroma R, et al. Dummy run and conformity indices in the ongoing EORTC low-grade glioma trial 22033-26033: first evaluation of quality of radiotherapy planning. Radiother Oncol. 2010;95(2):218-224.

15. Ali AN, Ogunleye T, Hardy CW, Shu HK, Curran WJ, Crocker IR. Improved hippocampal dose with reduced margin radiotherapy for glioblastoma multiforme. Radiat Oncol. 2014;9:20.

16. Liang BC, Thornton AF Jr, Sandler HM, Greenberg HS. Malignant astrocytomas: focal tumor recurrence after focal external beam radiation therapy. J Neurosurg. 1991;75(4):559-563.

17. Lee SW, Fraass BA, Marsh LH, et al. Patterns of failure following high-dose 3-D conformal radiotherapy for high-grade astrocytomas: a quantitative dosimetric study. Int J Radiat Oncol Biol Phys. 1999;43(1): 79-88.

18. Chan JL, Lee SW, Fraass BA, et al. Survival and failure patterns of high-grade gliomas after three-dimensional conformal radiotherapy. J Clin Oncol. 2002;20(6):1635-1642. patient perspectives such as quality of life, adherence and satisfaction. The manuscript management system is completely online and includes a very quick and fair peer-review system, which is all easy to use. Visit http://www.dovepress.com/testimonials.php to read real quotes from published authors. 